\title{
Formal Aspects of Grid Brokering*
}

\author{
Attila Kertész \\ MTA SZTAKI \\ 1518 Budapest, P.O. Box 63, Hungary \\ attila.kertesz@sztaki.hu
}

\author{
Zsolt Németh \\ MTA SZTAKI \\ 1518 Budapest, P.O. Box 63, Hungary \\ zsnemeth@sztaki.hu
}

\begin{abstract}
Coordination in distributed environments, like Grids, involves selecting the most appropriate services, resources or compositions to carry out the planned activities. Such functionalities appear at various levels of the infrastructure and in various means forming a blurry domain, where it is hard to see how the participating components are related and what their relevant properties are. In this paper we focus on a subset of these problems: resource brokering in Grid middleware. This paper aims at establishing a semantical model for brokering and related activities by defining brokering agents at three levels of the Grid middleware for resource, host and broker selection. The main contribution of this paper is the definition and decomposition of different brokering components in Grids by providing a formal model using Abstract State Machines.
\end{abstract}

\section{Introduction}

Grid Computing has emerged from the area of Parallel and Distributed Computing more than a decade ago [14]. Since then numerous Grid projects have succeeded and Grids started to flourish. Recently several service Grid middleware solutions are used around the world [6, 7, 18], and a wide variety of resource management tools - which are important components of grid computing infrastructure - are available and under development [15]. Resource management in Grids has many aspects and involves different approaches and means. Among those we have investigated brokering and established a Grid resource brokering taxonomy [12] to determine what properties brokers posses and what functionalities are desired for certain tasks. This survey shows that the currently available Grid resource management tools are built on different middleware components supporting different properties and named with a bunch of acronyms - even the ones having similar purposes. This plethora of approaches formed the domain of Grid resource management into a grey box with blurry boundaries where neither the users nor the researchers can clearly see how these tools are related and what their relevant properties are. Until the definitions and interrelations are clarified, further development and interoperability cannot be facilitated. Therefore, in an earlier work we aimed at an informal definition as Grid resource management anatomy in [13]. Present work can be considered as a continuation, investigating how the area of Grid resource management can be formalized and what essential layers, functionalities can be separated based on the formal model.

A former work this paper is built on presents a formal definition for Grid Computing [16]. That time there had been several definitions for Grid Computing without the ability of making a clear distinction between Grids and other distributed systems. The paper concluded that Grids cannot be defined purely by their properties rather, their runtime semantics make the real difference. Based on the analysis, a formal definition was given for Grid Computing revealing its essential and characteristic functionalities.

*The research leading to these results has received funding from the European Community's Seventh Framework Programme FP7/2007-2013 under grant agreement 215483 (S-Cube).

L. Brim and J. van de Pol (Eds.): 8th International Workshop on Parallel and Distributed Methods in verifiCation 2009 (PDMC'09)

EPTCS 14, 2009, pp. 18-31 doi 10.4204/EPTCS.14.2 (c) A. Kertész \& Zs. Németh

This work is licensed under the Creative Commons Attribution License. 
The aim and methodology of this paper is similar: establishing a formal, semantic model for Grid resource management using Abstract State Machine. We extend the formal model for Grids defined in [16] by classifying brokering components into three categories and defining three agents for resource management at different levels of Grid systems.

We are not aware of any other works that investigate formal models specifically for grid resource manager components. Bratosin et al. proposed a reference model for Grid architectures based on colored Petri nets in [4]. Though they provide a definition for job scheduling, they do not detail brokering steps and mechanisms at different levels. Altenhofen et al. investigated Service Oriented Architectures in [1], more specifically service discovery, mediation and composition. These components have some similar functionalities but this work is more focused on a unified, higher level service framework, and do not explore resource manager components. Börger et al. proposed an ASM model for workflows in [3]. The work presents workflow interpretations and transitions, which are related to our model, but they stay at the application level and do not deal with brokering at job level whereas our model targets the middleware below the application level.

In the following section we give a brief introduction of the formal Abstract State Machine method, and in Section 3 we summarize the formal model for Grid Computing introduced in [16] and describe our modified model. In Section 4 we present and describe the extensions for Grid brokering components and in Section 5 we refine agents responsible for broker and host selection. Finally Section 6 concludes the paper.

\section{Abstract State Machines}

ASM represents a mathematically well founded framework for system design and analysis [2]. It is able not just to model a working mechanism precisely but also to reveal the highly abstract nature of a system, i.e. to grasp the semantics [8]. Furthermore - unlike many other state based modeling methods -, it can easily be tailored to the required level of abstraction. Logicians structures applied in ASMs offer an expressive, flexible and complete way of state description. The basic sets and the functions interpreted on them can be freely chosen to the required level of complexity and precision [2].

In ASM, a signature (or vocabulary) is a finite set of function names, each of fixed arity. Furthermore, it also contains the symbols true, false, unde $f,=$ and the usual Boolean operators. A state $A$ of signature $\Upsilon$ is a nonempty set $X$ together with interpretations of function names in $\Upsilon$ on $X . X$ is called the superuniverse of $A$. An $r$-ary function name is interpreted as a function from $X^{r}$ to $X$, a basic function of $A$. A 0 -ary function name is interpreted as an element of $X$ [9]. A location of $A$ (can be seen like the address of a memory cell) is a pair $l=(f, a)$, where $f$ is a function name of arity $r$ in vocabulary $\Upsilon$ and $a$ is an $r$-tuple of elements of $X$. The element $f(a)$ is the content of location $l$. An update is a pair $a=(l$, $b$ ), where $l$ is a location and $b$ is an element of $X$. Firing $a$ at state $A$ means putting $b$ into the location $l$ while other locations remain intact. The resulting state is the sequel of $A$. It means that the interpretation of a function $f$ at argument $a$ has been modified resulting in a new state. ASMs are defined as a set of rules. An update rule $f(a):=b$ causes an update $((f, a), b)$, i.e. hence the interpretation of function $f$ on argument $a$ will result $b$. It must be emphasized that both $a$ and $b$ are evaluated in $A$.

The nullary Self function allows an agent to identify itself among other agents. It is interpreted differently by different agents (that is why it is not a member of the vocabulary.) An agent a interprets Self as $a$ while an other agent cannot interpret it as $a$. The Sel $f$ function cannot be the subject of updates. A conditional rule $R$ is of form 


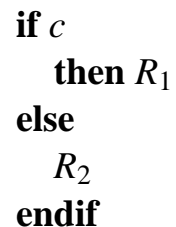

To fire $R$ the guard $c$ must be examined first and whenever it is true $R_{1}$ otherwise, $R_{2}$ must be fired. A block of rules is a rule and can be fired simultaneously if they are mutually consistent. Some applications may require additional space during their run therefore, the reserve of a state is the (infinite) source where new elements can be imported from by the following construct

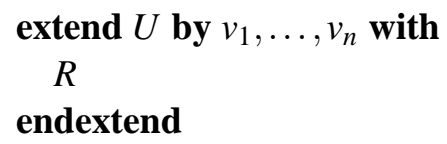

meaning that new elements are imported from the reserve and they are assigned to universe $U$ and then rule $R$ is fired [9].

The basic sequential ASM model can be extended in various ways like non-deterministic sequential models with the choice construct, first-order guard expressions, one-agent parallel and multi-agent distributed models. A distributed ASM [2] consists of a finite set of single-agent programs $\Pi_{n}$ called modules, a signature $\Upsilon$, which includes each $\operatorname{Fun}\left(\Pi_{\mathrm{n}}\right)-\{\operatorname{Sel} f\}$, i.e. it contains all the function names of each module but not the nullary Self function, and a collection of initial states.

As it can be seen, ASM states are represented as (modified) logicians structures, i.e. basic sets (universes) with functions interpreted on them. Structures are modified in ASM to enable state transitions for modeling dynamic systems. Applying a step of ASM $M$ to state (structure) $A$ will produce another state $A^{\prime}$ on the same set of function names. If the function names and arities are fixed, the only way of transforming a structure is to change the value of some functions for some arguments. Therefore, the most general structure transformation (ASM rule) is a guarded destructive assignment to functions at given arguments [2].

Refinement [2] is defined as a procedure where abstract and more concrete ASMs are related according to the hierarchical system design. At higher levels of abstraction implementation details have less importance whereas they become dominant as the level of abstraction is lowered giving rise to practical issues. Its goal is to find a controlled transition among design levels.

\section{ASM for Grid Computing}

Before we define our model, we summarize the ASM for Grids defined in [16]. Figure 1 (a) shows the important elements of this model. The ASM universes of the model are depicted on the left of the figures, and on the right a graphical representation of the connections of some elements of these universes and the most relevant functions governing process execution are shown. In this model user applications consist of one or more processes, while Grids consist of several nodes having one or more resources. During the execution of the user application first an agent maps the actual process of the application to a resource in the Grid, then the process is installed on the node of the resource as a task, which starts to use the resource. When all the processes of the application finished using their resources, the application is finished. 


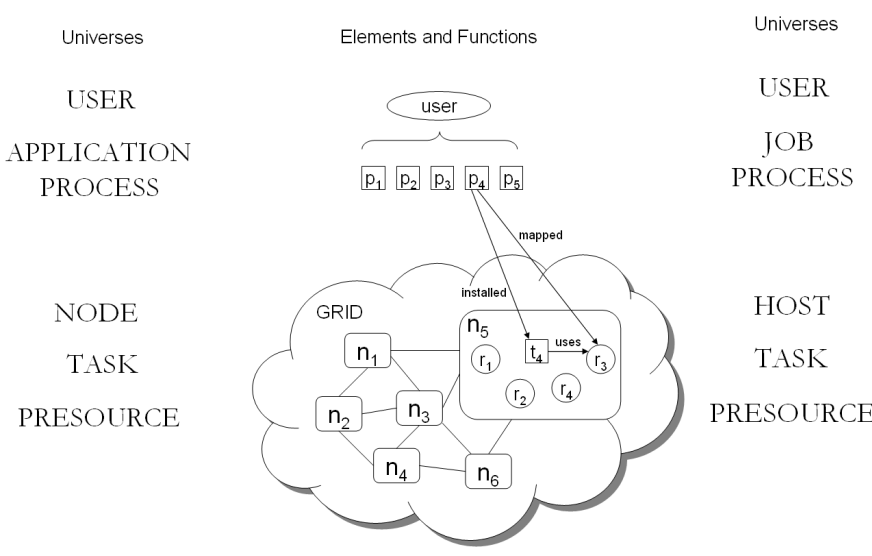

(a)

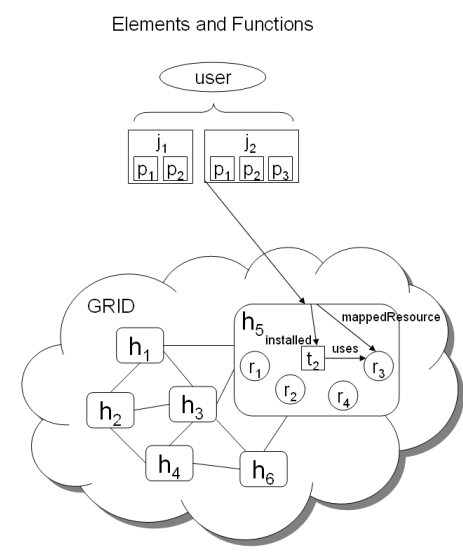

(b)

Figure 1: (a) Basic elements of the ASM model for Grids, and (b) the modified ASM model.

We extend this formal model by introducing Grid brokering at different levels. The basic model of grid systems introduced in [16] is presented in a slightly modified form here. The modification is indicated by introducing more practical issues related to realization; aligning the model to the terminology and naming conventions of grid brokering; and finally by experiences in Grid Computing since the paper was published. These modifications do not invalidate or alter the content and conclusion of the initial model just add more relevant details. The modifications are shown in Figure 1 (b). In the following subsections we define the basic elements of our proposed extended formal model based on ASM: the universes, the signature and the rules.

\subsection{Universes and signature}

To define our formal framework, first we need to examine real service Grid systems. Certain objects of the physical reality are modeled as elements of universes and relationships between real objects are represented as functions and relations. In Grid systems users (universe USER) define their applications in the form of jobs (universe $J O B$ ), which is the most typical computing paradigm for Grids hence, we restrict our model to this case. A job consists of one or more processes (universe PROCESS). The installed instances of processes are called as tasks (universe $T A S K$ ), which can be run on different hosts (universe HOST). Hosts are the building blocks of Grid systems, and typically a job is sent to a host for execution. A host may have several nodes (e.g. when a host is a cluster), and nodes have certain resources that processes require to run. Since nodes are usually invisible (and unmanageable) for higher level tools, therefore we neglect them in our model. In this way one or more physical resources (universe PRESOURCE) belong to a host, which also determines the physical location (universe LOCATION) of the resources. The processes of jobs require some of these resources to run. Users should select a host according to these resource requirements, which we call as abstract resources (universe ARESOURCE). Information on the physical resources of the hosts can be gathered by querying the information system of a Grid.

Once a job is submitted to a host, it is mapped to physical resources during execution. While a resource is busy, the mapped process is in waiting state. When the resource becomes free, the process starts using it and enters running state. Process termination implies a done state in case of successful run, and a failed state in case of an error. In general, Grid authorization allows users to log in to some 
hosts and validates user privileges to use some resources of some hosts [5]. The requested (abstract) and the physical resources have certain attributes (universe ATTR). Compatibility between an abstract and a physical resource means the physical resource can satisfy the process requirement. According to this informal description, the following functions are used in the model:

job: PROCESS $\rightarrow J O B$

user, globaluser, localuser: $J O B \rightarrow U S E R$

submitted: $J O B \times H O S T \rightarrow\{$ true, false $\}$

procRequest: PROCESS $\times$ ARESOURCE $\rightarrow\{$ true, false $\}$

uses: PROCESS $\times$ PRESOURCE $\rightarrow\{$ true false $\}$

mapped: PROCESS $\rightarrow$ LOCATION

belongsTo: PRESOURCE $\times$ HOST $\rightarrow\{$ true, false $\}$

installed: TASK $\times$ LOCATION $\rightarrow\{$ true, false $\}$

attr: $\{$ ARESOURCE, PRESOURCE $\} \rightarrow$ ATTR

location: PRESOURCE $\rightarrow$ LOCATION

handler: PRESOURCE $\rightarrow$ PROCESS

type: PRESOURCE $\rightarrow$ ATTR

compatible: $A T T R \times A T T R \rightarrow\{$ true, false $\}$

canLogin: $U S E R \times H O S T \rightarrow\{$ true, false $\}$

canUse: $U S E R \times$ PRESOURCE $\rightarrow\{$ true false $\}$

jobState: $J O B \rightarrow\{$ submitted, running, waiting, done, failed $\}$

procState: PROCESS $\rightarrow$ \{running, waiting $\}$

event: $T A S K \rightarrow\{$ start, abort, terminate $\}$

mappedHost: $J O B \rightarrow H O S T$

mappedResource: PROCESS $\times$ ARESOURCE $\rightarrow$ PRESOURCE

\subsection{Initial state}

We assume that $k$ processes belong to a job of a user. The job and its processes have some requirements, and no process and job is mapped to any resource or host. Therefore the states of the jobs and processes are undefined. In the following we define the initial state of our model:

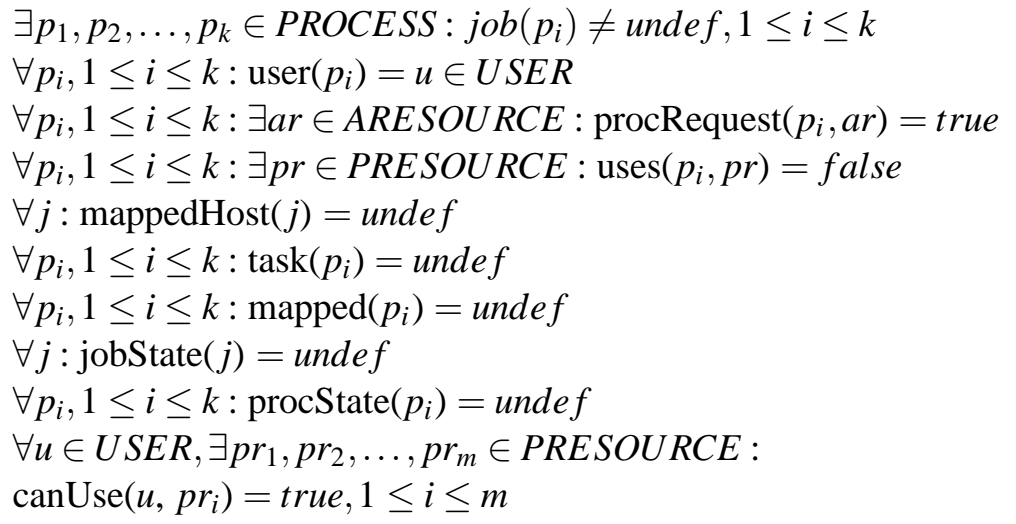

After we have defined the universes and the signature, in the following we give the rules of our model 
that constitute a module, i.e. a program that is executed by each agent in the model. The model presented here is a distributed multi-agent ASM where agents are jobs, i.e. elements from the $J O B$ universe. The working behavior of the brokering model is depicted from the perspective of the jobs hence, the self function is represented as $j$ and means the identity of a job, i.e. it can identify itself among other agents. It is interpreted differently by different agents.

\subsection{Rule 1: Resource selection}

According to Figure 1(b), when the job is sent to a host, the required resources need to be selected that are used by the processes of the job. During job execution, a task of each process is installed to the location of the required and selected resource. The precondition of resource selection is that the process of the job should be able to use the mapped resource. In case of the process can directly access the physical resource $\left(r_{d}\right)$ the execution (resource usage) is automatically started, otherwise a local handler process should provide the execution platform (i.e. the additional software or service). If this handler process does not exist, it should be started before execution. The agent responsible for resource mapping needs to ensure that the chosen resource fulfills the abstract resource requirement of the process. Here is the formal definition:

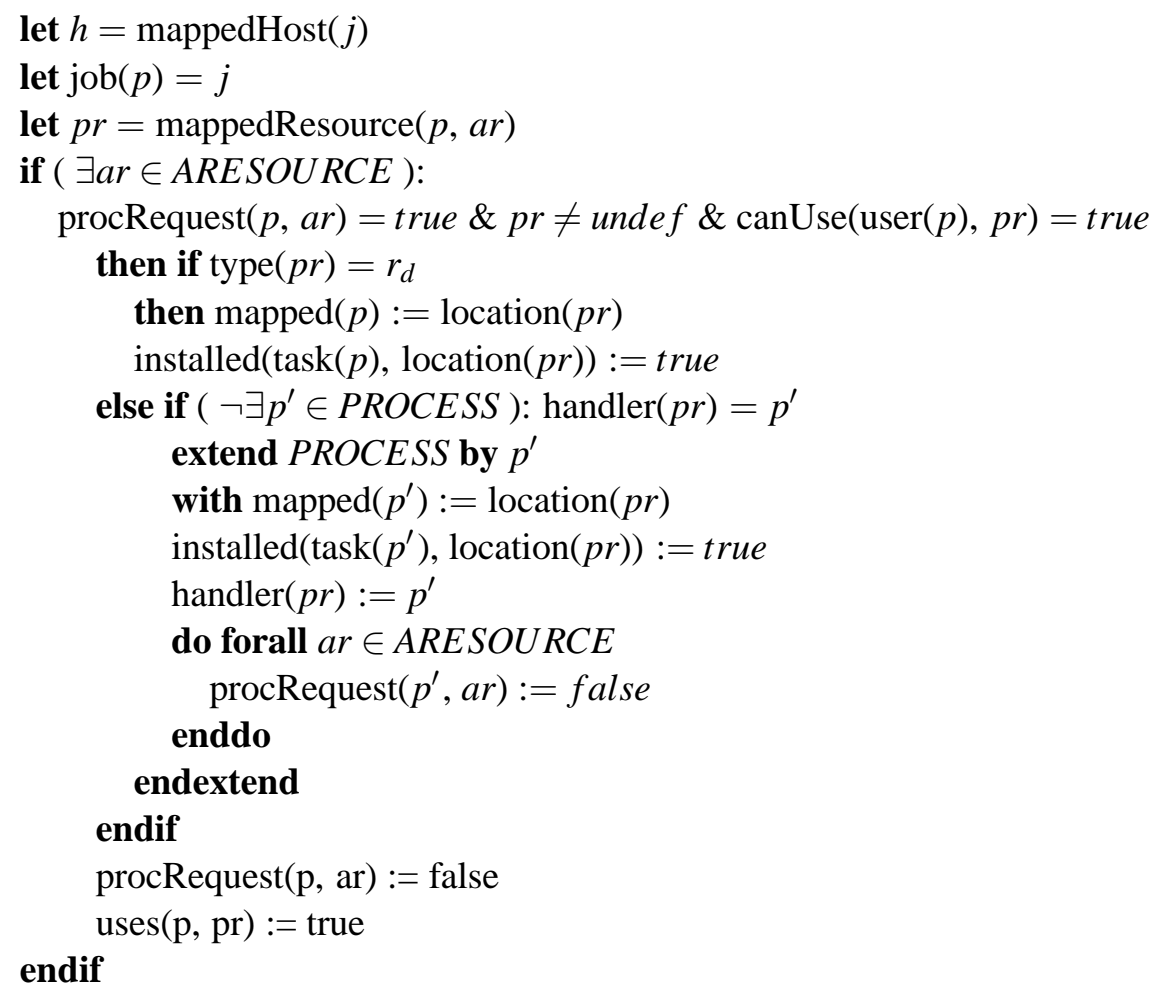

$\Pi_{\text {resource_mapping }}$

if ( $\exists a r \in A R E S O U R C E, \exists p \in P R O C E S S, \exists h \in H O S T$ ): $\operatorname{job}(p)=j \&$ mappedResource $(p$, ar $)=$ undef \& 


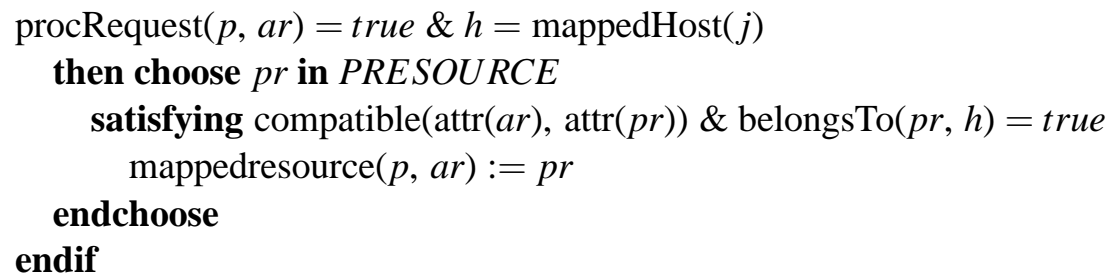

Here, we note that though generally a job runs on a host (if it is a parallel job of communicating processes, it runs on a number of nodes of this host parallelly), some middleware tools may enable coallocation of parallel processes on nodes of different hosts. We do not deal with this situation, since it is rarely used and supported, but further refinement of our model could represent such cases.

Before job execution it is necessary to authenticate users. In Service Grids users are authenticated by proxies of grid certificates [5]. A local process is responsible for validating these proxies by mapping global users to local ones having the same privileges. The related formalism of user mapping is similar to the one presented in [16].

\subsection{Rule 2: State transition}

In this subsection we define, how job states are evolving during execution:

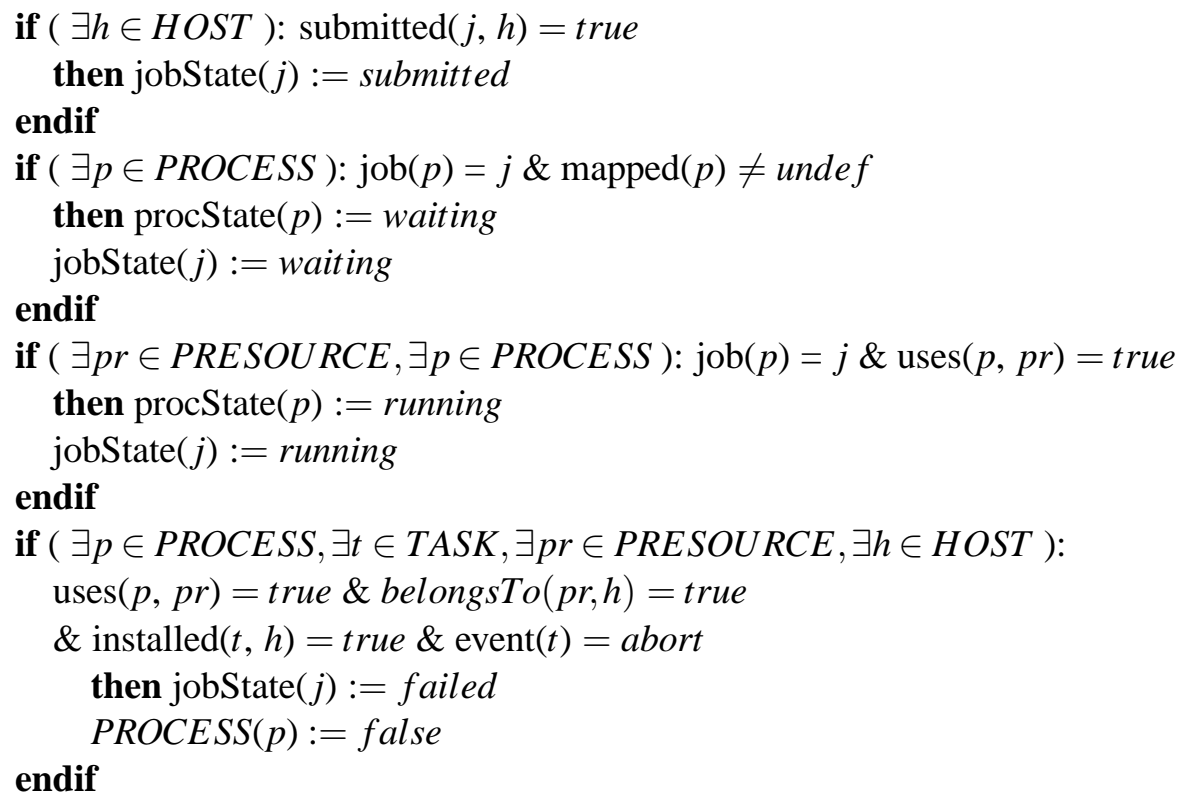

Though in general, process spawning could cause additional resource requests for job execution in a host, we do not detail this in our model, and keep it as abstract as possible, since at the level of grid brokering process communications and spawning are invisible. In order to handle these situations, we assume that resource requests of spawned processes are known a priori. State transitions related to job termination are formalized in Rule 3. 


\subsection{Rule 3: Termination}

Job execution is terminated under the following conditions:

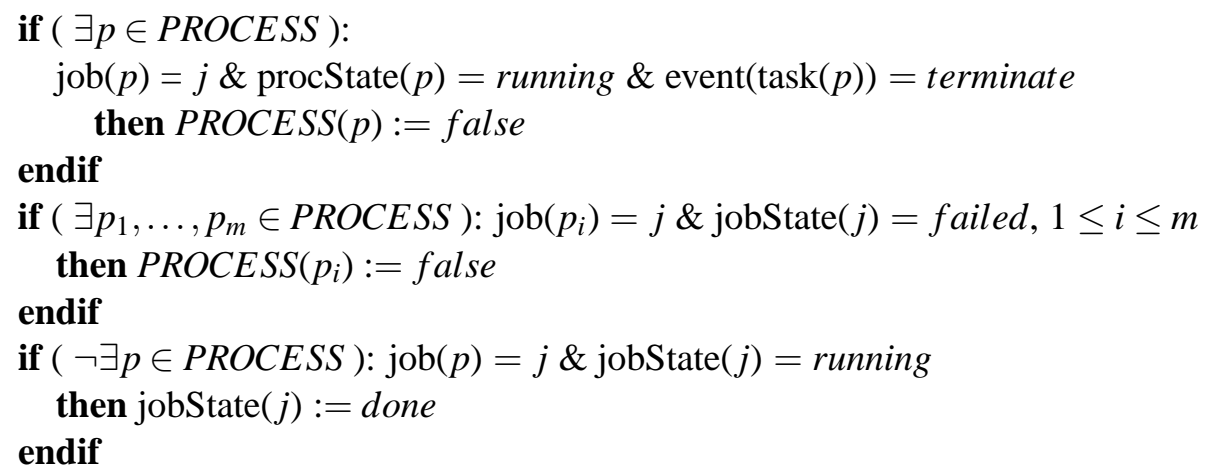

\section{ASM for Grid Brokering}

This section focuses on middleware components responsible for brokering in Grids. In our ASM model these components are represented by agents (also called as modules). First we give an informal overview of these components and their roles in Grids and demonstrate their usage in a typical Grid application execution scenario. In the following subsections we show how these components can appear as agents in the formal model described above. Furthermore we emphasize how these brokering components contribute to Grid Interoperability, i.e. how they support transparent job submissions to different, separated Grids.

At the lowest level of Grid resource management we can find local resource managers (also called as schedulers or cluster managers, e.g. PBS [17]) that were taken from high-performance and distributed computing, and now generally used in Grid Systems. Their goal is to schedule and manage single and parallel programs and their processes locally. This local management is formalized in Rule 1 of or model. In this case interoperability is not supported at all. Without additional brokering components users need to choose from the available hosts manually relying on mostly static information.

One level above, a grid Resource Management System (RMS), also called as a Grid resource broker, is needed to automate host selection. It can be an internal middleware service, or an external tool that uses other middleware components or services. (Note that the word "resource" is used differently in our model as in the expression "resource broker". In our model we call the computing and storage elements of Grids as "hosts", and "resources" are the physical components of the hosts, e.g. processor and memory.) While local managers usually schedule user programs in a grid host (e.g. in a cluster) among free processors, the Grid broker schedules jobs at the level of Grid middleware by selecting a host that best matches the requirements of these jobs. (Thus, the selected host can also be a cluster with a local manager.) Therefore, a broker is also called as a meta-scheduler - more information on broker naming conventions and their connections can be found in [13]. Some of them support different middleware solutions, job types, agreements or various quality of service (QoS) attributes. Furthermore different brokers may be connected to different hosts and Grids. A taxonomy in [12] introduces these properties and shows the differences among the currently used brokers, their properties, organization and connections and among their level of interoperability. In our future work we also plan to represent interoperability as a metric in 
our model in order to categorize and differentiate various brokering components.

With the help of grid brokers, host selection is automated, but users are still bound to separate grid islands (i.e. grid systems that are complete systems on their own but closed to any form of interoperability between each other, either by technology, compatibility, administrative or other restrictions) managed by their own brokers. Nevertheless users have the ability to select manually, which broker and Grid they would like to use (even static information on broker properties are available in form of manuals or taxonomies e.g. in [12]). In order to achieve the highest level of interoperability broker selection should also be automated. Therefore at the highest level we can find meta-brokering [11], which is a novel approach that introduces another layer above current grid brokers in order to facilitate inter-grid load balancing and interoperable brokering. The Grid Meta-broker sits on top of Grid brokers and uses metadata to decide which broker should be selected for a user's job. To demonstrate the interoperation of these brokering components, we describe a typical Grid usage scenario for a job execution that requires the following steps:

1. The user defines its application as jobs, also stating the requirements of its execution.

2. The user requirements of the job is examined by the meta-broker, and mapped to the properties of the available brokers. A proper broker, that is able to submit the job, is selected for submission.

3. The selected broker examines the resource requirements of the job and matches them to the physical resources of the available hosts. A host having all the required resources is selected for execution.

4. The agent on the selected host (the local resource manager) maps the resource requirements of the job to the available physical resources during execution.

In the following subsections we define two more rules to model the informal description and discussion above. We need additional universes and functions to incorporate brokering into our model.

\subsection{An additional rule for Grid Brokering}

Brokers (universe BROKER) are responsible for host selection, therefore hosts are managed by brokers, which can have different properties (universe PRORERTY) that users may require for job execution. A user should select a broker for its job according to these requirements (universe REQUIREMENT). Furthermore we place universe ARESOURCE as a subset of universe REQUIREMENT, since the elements of both sets represent user requirements, and universe PRESOURCE can be a subset of universe PRORERTY, because physical resources can be regarded as host properties. The following functions are added to the model:

request: $J O B \times R E Q U I R E M E N T \rightarrow\{$ true, false $\}$

submitted: $J O B \times\{H O S T, B R O K E R\} \rightarrow\{$ true, false $\}$

manages: $H O S T \times B R O K E R \rightarrow\{$ true, false $\}$

have: $B R O K E R \times P R O R E R T Y \rightarrow\{$ true, false $\}$

attr: $\{$ REQUIREMENT, PRORERTY $\} \rightarrow$ ATTR

We extend the initial state by:

$\forall j: \exists r \in R E Q U I R E M E N T: \operatorname{request}(j, r)=$ true 


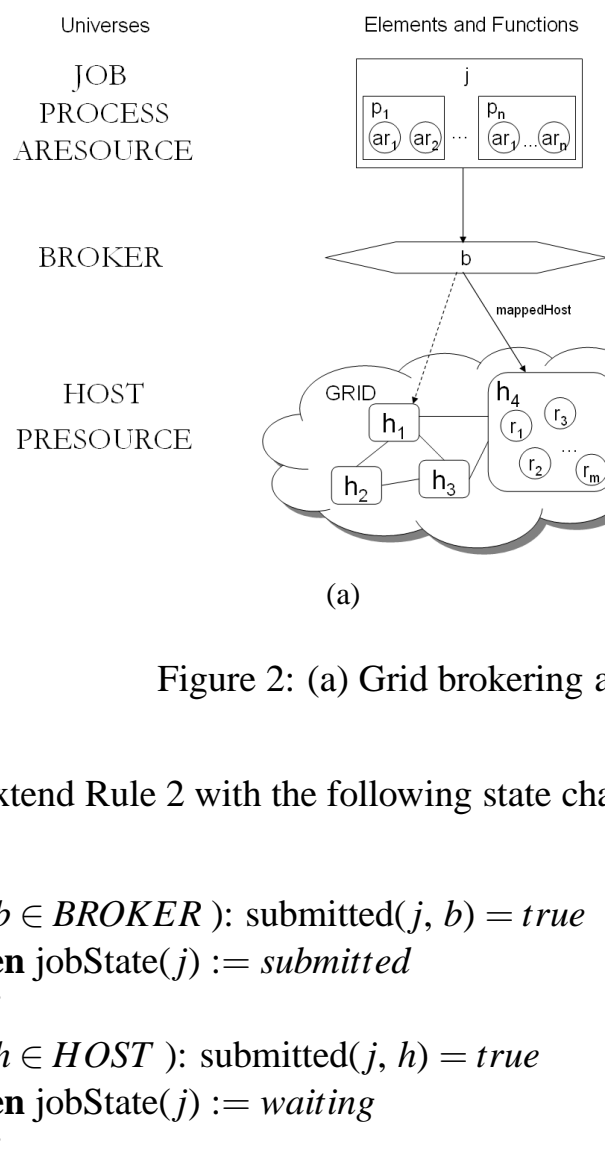

Once a broker is selected by the user, it should find an execution host. The precondition of this host selection process is that the user of the job should be able to use the required resources of the selected host. The broker agent responsible for host mapping needs to ensure that the chosen host has all the resources requested by the processes of the job. This additional component responsible for Grid brokering is highlighted in Figure 2 (a). In the following we state the formal definition:

Rule 4: Host selection

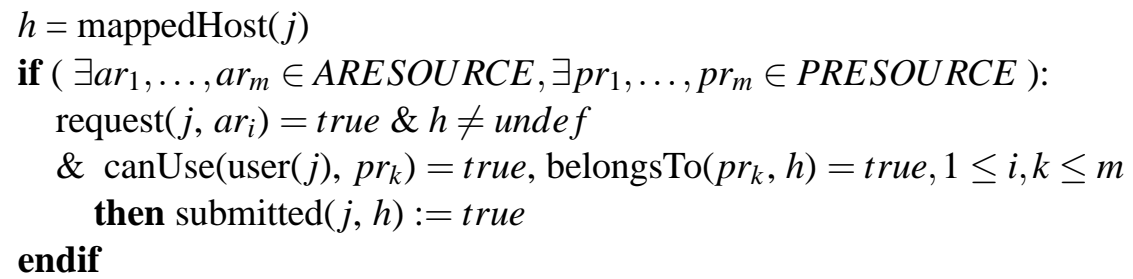

$\Pi_{\text {host_mapping }}$

if $\left(\exists j \in J O B, \exists a r_{1}, \ldots, a r_{m} \in A R E S O U R C E, \exists p r_{1}, \ldots, p r_{m} \in P R E S O U R C E\right)$ : 


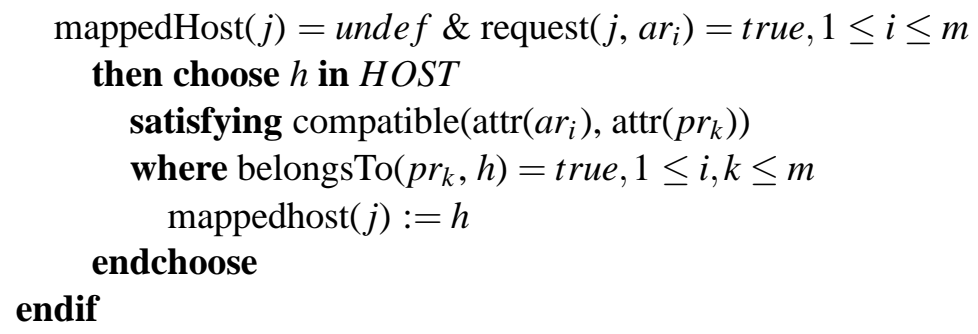

\subsection{Rule 5: Broker selection}

At the highest level of Grid resource management a broker needs to be selected automatically for a user job. An important precondition of the selection process is that such a broker needs to be selected that manages hosts with resources that the user of the job can use. Furthermore the agent responsible for broker selection, the meta-broker (universe METABROKER) needs to ensure that the chosen broker has all the properties required by the user's job. Therefore users need to characterize their job requirements in a certain job description language, which should include both the required broker properties and abstract resources of the processes of the job. This additional Grid middleware component is highlighted in Figure 2 (b). The following function is added to the model:

mappedBroker: $J O B \rightarrow B R O K E R$

We extend the initial state by:

$\forall j:$ mappedBroker $(j)=$ undef

The formal definition of the meta-broker agent is as follows:

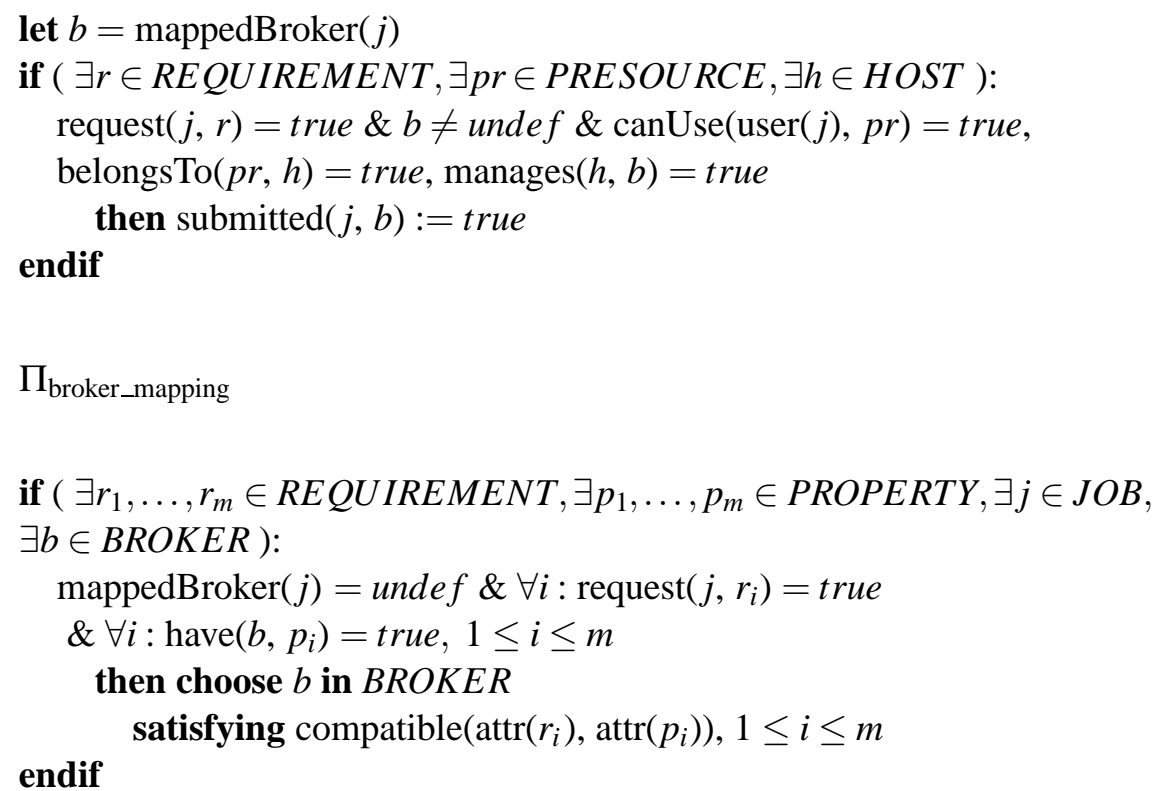


Finally we should state that jobs can be interconnected in order to form a complex Grid application called as workflows. The execution of workflows require a coordinating tool called workflow enactor that schedules the interdependent jobs for executions. We refrain from formalizing workflow management and incorporate it into our model, since our central entities are jobs, and therefore assume that grid applications are submitted into the system in the form of jobs.

As a summary, we have shown that grid brokering takes place at three levels, and the following operations need to be performed: broker mapping, host mapping and resource mapping. In the following section we show, how practical examples of these components can be described by our formal ASM model with the help of ASM refinement. These tools are the Grid Meta-Broker [11] and GTbroker [10].

\section{Refinement of Broker Components}

This section contains illustrative examples, how the generic brokering model can be refined into models that represent realised implementations of the brokering principles. One can see in these examples how certain functions, kept abstract in Rule 4 and 5 presented earlier, are transformed to reveal implementation details. More information on the realization and practical utilization of these tools can be read in [10].

\subsection{Refinement of broker mapping (matchmaking of the Grid Meta-Broker)}

$\Pi_{\text {broker_mapping }}^{\prime}$

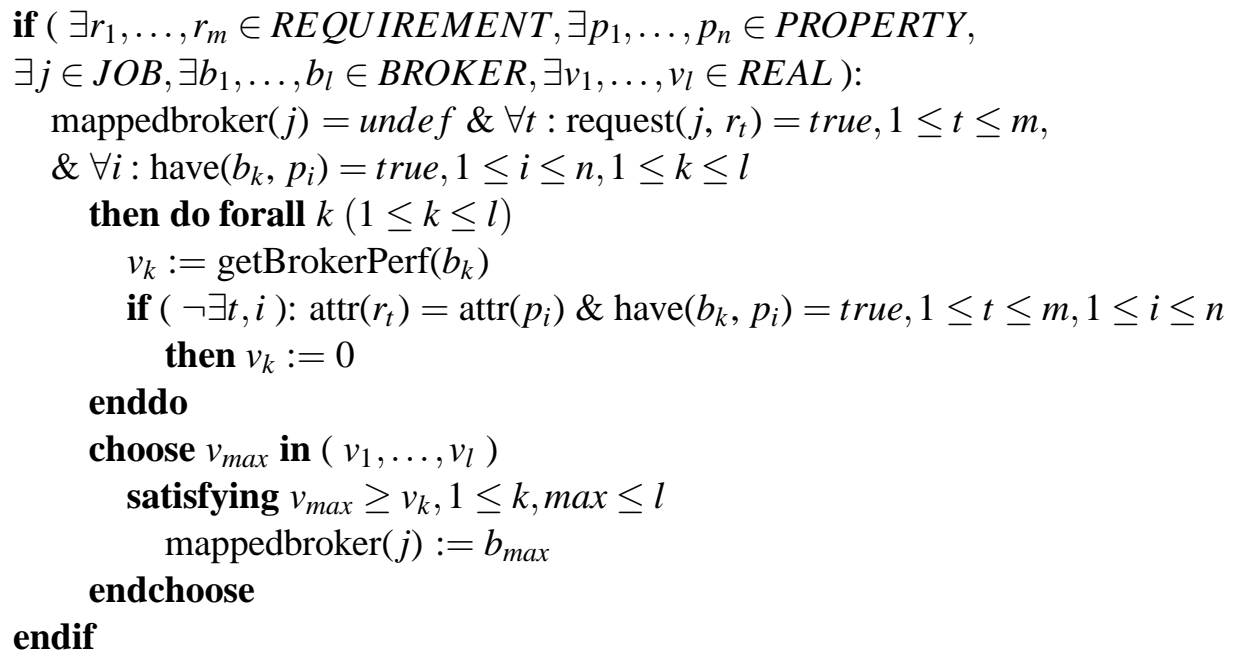

In addition to the broker mapping defined in Rule 1, this refinement details how the compatible function is implemented. In case of the Grid Meta-broker, the attributes of the broker properties are certain keywords. The users have to use the same keywords in their requirement specifications, therefore compatibility means exact string matching. The refined agent also uses an additional function getBrokerPerf: $B R O K E R \rightarrow R E A L$, which returns a real number denoting the dynamic performance of the appropriate broker. The higher this value is the better the broker performs. 


\subsection{Refinement of host mapping (matchmaking of GTbroker)}

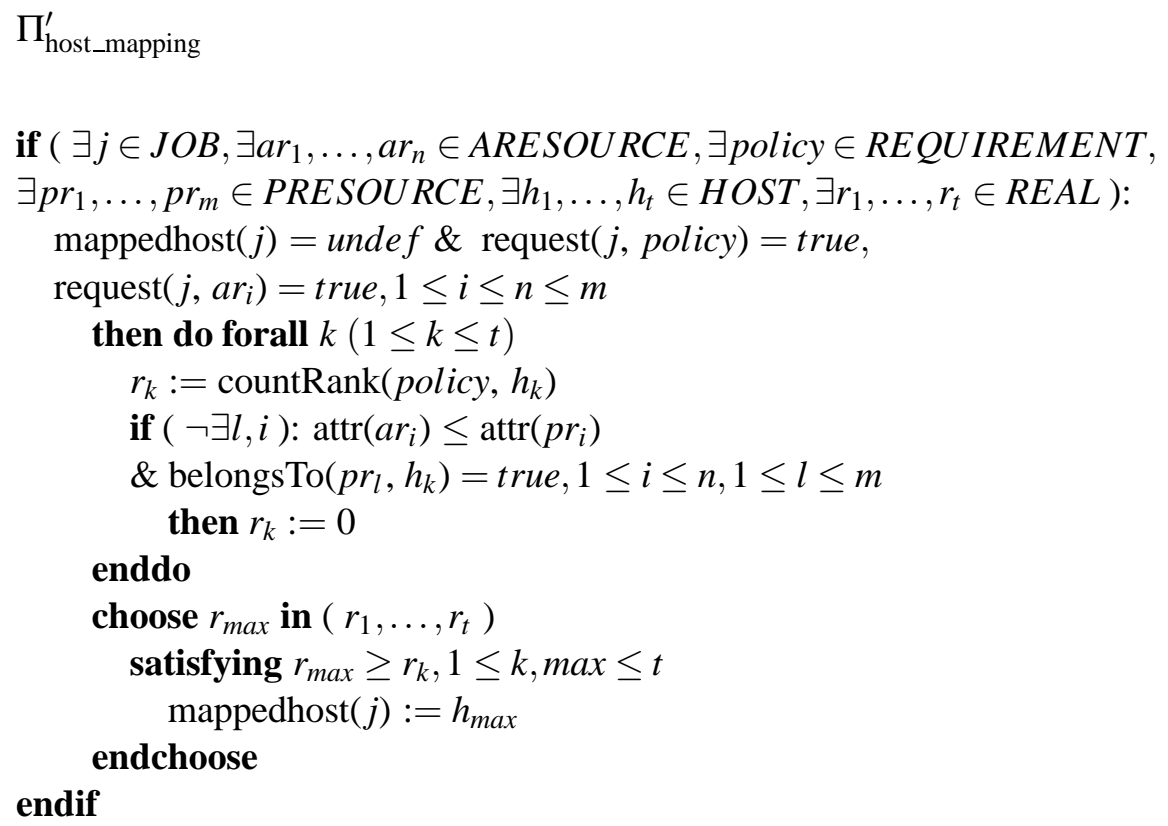

In addition to the host mapping defined in Rule 2, this refinement also reveals the meaning of the compatible function. In case of GTbroker, the attributes of resource requirements denote the amount of resource capacity (e.g. memory size or processor speed) needed by the processes of the job for execution. This means, if the available physical resource has equal or greater capacity than requested, the process can run. The host selection method can be influenced by users using the special policy requirement. The value of its attribute tells the additional countRank: REQUIREMENT $\times H O S T \rightarrow R E A L$ function how to compute the rank for the available hosts (e.g. higher priority can be given to hosts with faster processors). Finally, the host with the highest rank is selected for execution.

\section{Conclusions}

In this paper we have investigated the brokering components of the Grid middleware and defined a three-layered formal model using Abstract State Machines. In this model three agents are responsible for resource management by performing three selection processes: broker mapping, host mapping and resource mapping. We have also proposed two refined definitions for broker and host selection, which are implemented by the Grid Meta-broker and GTbroker. Our future work aims at introducing interoperability metrics for categorizing brokering components and using the ASM model for verifying our categorization.

\section{References}

[1] Altenhofen M., A. Friesen and J. Lemcke (2008): ASMs in Service Oriented Architectures. Journal of Universal Computer Science, vol. 14, no. 12, pp. 2034-2058.

[2] Börger, E., and R. Stark (2003): Abstract State Machines. A method for High-level System Design and Analysis. Springer. 
[3] Börger, E. and B. Thalheim (2008): Modeling Workflows, Interaction Patterns, Web Services and Business Processes: The ASM-Based Approach. In Proceedings of the 1st international Conference on Abstract State Machines, B and Z, Lecture Notes In Computer Science, vol. 5238. Springer-Verlag, pp. 24-38.

[4] Bratosin, C., W. Aalst, N. Sidorova, and N. Trcka (2008): A Reference Model for Grid Architectures and Its Analysis. In Proceedings of the OTM 2008 Confederated international Conferences, Lecture Notes In Computer Science, vol. 5331. Springer-Verlag, pp. 898-913.

[5] Foster, I., C. Kesselman, G. Tsudik, and S. Tuecke (1998): A security architecture for computational grids. In Proceedings of the 5th ACM Conference on Computer and Communications Security, ACM, pp. 83-92.

[6] gLite middleware for grid computing, Available at http://glite.web.cern.ch/glite/.

[7] Globus Toolkit, Available at http://www.globus.org/toolkit/.

[8] Gurevich, Y. (1993): Evolving Algebras: An Attempt to Discover Semantics. Current Trends in Theoretical Computer Science, pp. 266-292.

[9] Gurevich, Y. (1997): Draft of the ASM Guide, Available at http://www.eecs .umich.edu/gasm/papers/ guide97.html.

[10] Kertész, A., and P. Kacsuk (2009): Grid Interoperability Solutions in Grid Resource Management. IEEE Systems Journal, vol. 3, Special Issue on Grid Resource Management, pp. 131-141.

[11] Kertész, A., and P. Kacsuk (2009): GMBS: A new middleware service for making grids interoperable. Future Generation Computer Systems, doi:10.1016/j.future.2009.10.007.

[12] Kertész, A., and P. Kacsuk (2007): A Taxonomy of Grid Resource Brokers. 6th Austrian-Hungarian Workshop on Distributed and Parallel Systems (DAPSYS 2006), Springer US, pp. 201-210.

[13] Kertész, A., and T. Prokosch (2010): The Anatomy of Grid Resource Management. Remote Instrumentation and Virtual Laboratories, Service Architecture and Networking, Springer US, to be appear.

[14] Kesselman, C., and I. Foster (1998): The Grid: Blueprint for a New Computing Infrastructure. Morgan Kaufmann Publishers.

[15] Krauter, K., R. Buyya, and M. Maheswaran (2002): A taxonomy and survey of grid resource management systems for distributed computing. Softw., Pract. Exper., vol. 32, pp. 135-164.

[16] Németh, Zs., and V. Sunderam (2003): Characterizing Grids: Attributes, Definitions, and Formalisms. Journal of Grid Computing, vol. 1, pp. 9-23.

[17] PBS GridWorks, Available at http://www.pbsgridworks.com/.

[18] Uniform Interface to Computing Resources (UNICORE), Available at http://www .unicore.eu/. 